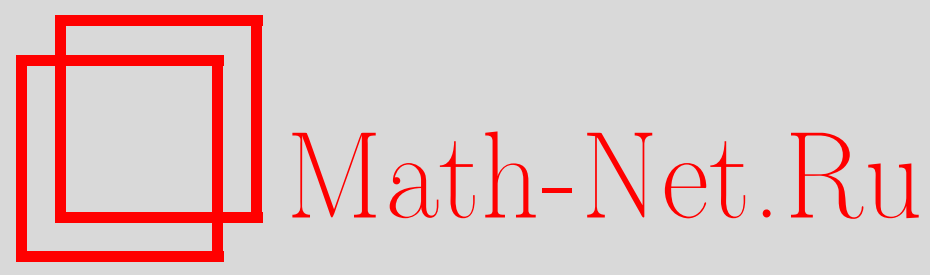

В. В. Курта, K вопросу об отсутствии положительных решений у эллиптических уравнений, Матем. заметки, 1999, том 65, выпуск 4, 552-561

DOI: https://doi.org/10.4213/mzm1081

Использование Общероссийского математического портала Math-Net.Ru подразумевает, что вы прочитали и согласны с пользовательским соглашением http://www.mathnet.ru/rus/agreement

Параметры загрузки:

IP : 54.196 .121 .252

26 апреля 2023 г., $17: 56: 28$

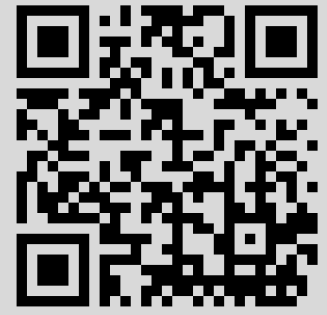




\section{К ВОПРОСУ ОБ ОТСУТСТВИИ ПОЛОЖИТЕЛЬНЫХ РЕШЕНИЙ У ЭЛЛИПТИЧЕСКИХ УРАВНЕНИЙ}

\section{В.В. Курта}

Основньм результатом работы являются утверждения об отсутствии целых положительных решений в шшроких классах квазилинейньх эллиптических уравнений, модельньми примерами которых являются уравнения вида

$$
\sum_{i=1}^{n} \frac{\partial}{\partial x_{i}}\left(|\nabla u|^{\alpha-2} u_{x_{i}}\right)=-|u|^{q-1} u
$$

где $\alpha>1$ и $q$ - некоторые фиксированные действительные числа.

Библиографоия: 13 названий.

Основным результатом работы являются утверждения об отсутствии целых положительных решений в широких классах квазилинейных эллиптических уравнений, модельными примерами которых являются уравнения вида

$$
\begin{aligned}
\sum_{i=1}^{n} \frac{\partial}{\partial x_{i}}\left(|\nabla u|^{\alpha-2} u_{x_{i}}\right) & =-|u|^{q-1} u \\
\sum_{i=1}^{n} \frac{\partial}{\partial x_{i}}\left(\left|u_{x_{i}}\right|^{\alpha-2} u_{x_{i}}\right) & =-|u|^{q-1} u \\
\sum_{i=1}^{n} \frac{\partial}{\partial x_{i}}\left(\frac{|\nabla u|^{\alpha-2} u_{x_{i}}}{\sqrt{1+|\nabla u|^{2}}}\right) & =-|u|^{q-1} u
\end{aligned}
$$

где $\alpha>1$ и $q$ - некоторые фиксированные действительные числа.

Заметим, что уравнение вида

$$
\Delta u=-|u|^{q-1} u
$$

является частньм случаем уравнений вида $(1),(2)$ при $\alpha=2$.

Утверждения об отсутствии тех или иных решений у уравнений вида (1)-(4) играют важную роль в теории и приложениях. Однако, многочисленные результаты об отсутствии целых решений в рассматриваемых классах уравнений (см., например, [1]-[6] и цитируемую там литературу), как правило, установлены только для гладких решений, зачастую обремененных еще и свойством радиальной симметрии.

Работа выполнена при финансовой поддержке Российского фонда фундаментальых исследований, грант № 96-01-00097, и фонда INTAS, грант № 96-1060. 
Целью данной статьи является изложение результатов, полученных автором в [7], [8] и частично анонсированных в [9], об отсутствии целых положительных локально ограниченных решений в широких классах квазилинейньх эллиптических уравнений, принадлежащих лишь локально соответствуюшим пространствам Соболева без предположения какой-либо симметрии.

Обозначим через $L$ дифференциальньй оператор, формально определяемьй равенстBOM

$$
L u=\sum_{i=1}^{n} \frac{d}{d x_{i}} A_{i}(x, u, \nabla u),
$$

где $A_{i}(x, \eta, \xi), i=1, \ldots, n, n \geqslant 2,-$ каратеодориевы на множестве $\mathbb{R}^{n} \times \mathbb{R}^{1} \times \mathbb{R}^{n}$ функции.

ОПРЕДЕЛЕНИЕ. Пусть $\alpha \geqslant 1$ - произвольное фиксированное число. Будем говорить, что оператор $L$, определенньй соотношением (5), принадлежит классу $A(\alpha)$, если существует положительная постоянная $\mathscr{K}$ такая, что для почти всех $x \in \mathbb{R}^{n}$, всех $\eta \in \mathbb{R}^{1}$ и всех $\xi, \psi \in \mathbb{R}^{n}$ выполнены неравенства

$$
\begin{aligned}
0 & \leqslant \sum_{i=1}^{n} \xi_{i} A_{i}(x, \eta, \xi), \\
\left|\sum_{i=1}^{n} \psi_{i} A_{i}(x, \eta, \xi)\right|^{\alpha} & \leqslant \mathscr{K}|\psi|^{\alpha}\left(\sum_{i=1}^{n} \xi_{i} A_{i}(x, \eta, \xi)\right)^{\alpha-1} .
\end{aligned}
$$

Очевидно, что одним из достаточных условий вьполнимости (7) является выполнимость неравенства

$$
\left(\sum_{i=1}^{n} A_{i}^{2}(x, \eta, \xi)\right)^{\alpha / 2} \leqslant \mathscr{K}\left(\sum_{i=1}^{n} \xi_{i} A_{i}(x, \eta, \xi)\right)^{\alpha-1}
$$

Легко проверяется, что дифференциальные операторы, стоящие слева в уравнениях (1)-(3), принадлежат классу $A(\alpha)$ при соответствуюшем фиксированном $\alpha>1$.

Ограничения на рост дифференциальных операторов $L$ в виде неравенств (6)-(8), введенные В. М. Миклюковым [10], [11], обладают рядом преимуществ по сравнению с традиционными

$$
|A(x, \eta, \xi)| \leqslant \nu_{1}|\xi|^{\alpha-1} \quad \text { и } \quad|(\xi \times A(x, \eta, \xi))| \geqslant \nu_{2}|\xi|^{\alpha},
$$

где $\nu_{1}, \nu_{2}-$ произвольные положительные фиксированные постоянные. Основное из них условия (6)-(8) менее ограничительны. Приведем соответствуюшие примеры.

Пусть $a(x, \eta, \xi)$ - произвольная достаточно гладкая, неотрицательная, равномерно ограниченная функция, определенная на множестве $\mathbb{R}^{n} \times \mathbb{R}^{1} \times \mathbb{R}^{n}$. Нетрудно проверить, что дифференциальный оператор, формально определяемьй равенством

$$
N u=\sum_{i=1}^{n} \frac{\partial}{\partial x_{i}}\left(a(x, u, \nabla u)|\nabla u|^{\alpha-2} u_{x_{i}}\right)
$$

принадлежит классу $A(\alpha)$ при соответствующем параметре $\alpha$ и не удовлетворяет, вообще говоря, условиям (9) ни при каком фиксированном $\alpha>1$. 
Может оказаться, что оператор $L$, определяемьй соотношением (5), принадлежит нескольким классам $A(\alpha)$. Например, дифференциальньй оператор $L$ из уравнения (3) принадлежит классам $A(\alpha-1)$ и $A(\alpha)$ при любом фиксированном $\alpha \geqslant 2$.

То же относится к хорошо известному оператору средней кривизны

$$
L u=\sum_{i=1}^{n} \frac{\partial}{\partial x_{i}}\left(\frac{u_{x_{i}}}{\sqrt{1+|\nabla u|^{2}}}\right)
$$

который, например, принадлежит классам $A(1)$ и $A(2)$. Заметим, кстати, что коэффициенты этого оператора не удовлетворяют соотношениям (9) при $\alpha=1$ и $\alpha=2$.

Остановимся более подробно на классе $A(2)$. Пусть $L$ - оператор, формально определяемьй соотношением вида

$$
L u=\sum_{i, j=1}^{n} \frac{\partial}{\partial x_{i}}\left(a_{i j}(x, u, \nabla u) \frac{\partial u}{\partial x_{j}}\right),
$$

где $a_{i j}(x, \eta, \xi)$ - равномерно ограниченные и каратеодориевы на множестве $\mathbb{R}^{n} \times \mathbb{R}^{1} \times \mathbb{R}^{n}$ функции такие, что $a_{i j}(x, \eta, \xi)=a_{j i}(x, \eta, \xi)$ и

$$
0 \leqslant \sum_{i, j=1}^{n} a_{i j}(x, \eta, \xi) \psi_{i} \psi_{j}
$$

для почти всех $x \in \mathbb{R}^{n}$, всех $\eta \in \mathbb{R}^{1}$ и всех $\xi, \psi \in \mathbb{R}^{n}$.

Заметим, что частным случаем оператора $L$ является линейный дивергентный, вообще говоря, вырождающийся эллиптический оператор второго порядка.

Покажем, что оператор $L$ принадлежит классу $A(2)$. Для этого положим

$$
A_{i}(x, \eta, \xi)=\sum_{j=1}^{n} a_{i j}(x, \eta, \xi) \xi_{j}, \quad i=1, \ldots, n
$$

и проверим, что дифференпиальньй оператор $L$, формально определяемый соотношением (5) с коэффициентами из (11), удовлетворяет условиям (6), (7) при $\alpha=2$.

Условие (6) проверяется тривиально, поскольку в этом случае оно имеет простой вид:

$$
\sum_{i=1}^{n} \xi_{i} A_{i}(x, \eta, \xi)=\sum_{i, j=1}^{n} a_{i j}(x, \eta, \xi) \xi_{i} \xi_{j}
$$

Проверим вьполнимость условия (7) при $\alpha=2$. Поскольку

$$
\sum_{i=1}^{n} \psi_{i} A_{i}(x, \eta, \xi)=\sum_{i, j=1}^{n} a_{i j}(x, \eta, \xi) \psi_{i} \xi_{j}
$$

то, оценивая правую часть этого тождества по неравенству Коши, имеем

$$
\left(\sum_{i=1}^{n} \psi_{i} A_{i}(x, \eta, \xi)\right)^{2} \leqslant \sum_{i, j=1}^{n} a_{i j}(x, \eta, \xi) \xi_{i} \xi_{j} \sum_{i, j=1}^{n} a_{i j}(x, \eta, \xi) \psi_{i} \psi_{j} .
$$


Используя условие ограниченности коэффициентов матрицы $\left\{a_{i j}(x, \eta, \xi)\right\}$, получаем, что

$$
\left(\sum_{i=1}^{n} \psi_{i} A_{i}(x, \eta, \xi)\right)^{2} \leqslant \mathscr{K}|\psi|^{2} \sum_{i=1}^{n} \xi_{i} A_{i}(x, \eta, \xi)
$$

для почти всех $x \in \mathbb{R}^{n}$, всех $\eta \in \mathbb{R}^{1}$, всех $\xi, \psi \in \mathbb{R}^{n}$ и некоторого неотрицательного $\mathscr{K}$.

Следовательно, дифференциальньй оператор $L$, определенньй соотношением вида $(10)$, является элементом класса $A(2)$ и не удовлетворяет, вообше говоря, условиям (9). Аналогично обстоит дело с линейным дивергентным эллиптическим оператором, не удовлетворяющим условию равномерной эллиптичности, а именно: он является элементом класса $A(2)$ и не удовлетворяет условиям (9).

В работе изучаются уравнения вида

$$
L u=-f(u)
$$

где оператор $L$ принадлежит классу $A(\alpha)$ при некотором фиксированном $\alpha \geqslant 1$, а функция $f(u)=a|u|^{q-1} u$ при некоторых действительных $a>0$ и $q$.

Определим обобщенное целое положительное (неотрицательное) суперрешение уравнения вида (12).

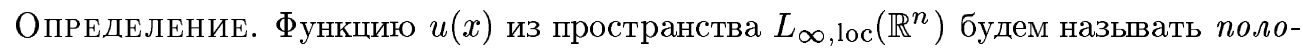
жительной (неотричательной) в $\mathbb{R}^{n}$, если $\operatorname{ess} \inf u(x)$, взятый по любому шару в $\mathbb{R}^{n}$, положителен (неотрицателен).

ОПРЕДЕЛЕНИЕ. Пусть $\alpha \geqslant 1$ и $q$ - произвольные фиксированные действительные числа, оператор $L$ принадлежит классу $A(\alpha)$. Функцию $u(x)$ будем называть обобщенным иелым положсительным суперрешением уравнения вида (12), если она принадлежит пространству $W_{\alpha, \text { loc }}^{1}\left(\mathbb{R}^{n}\right) \cap L_{\infty, \text { loc }}\left(\mathbb{R}^{n}\right)$, положительна и для произвольной неотрицательной функции $\varphi$ из пространства $\stackrel{\circ}{W}_{\alpha}^{1}\left(\mathbb{R}^{n}\right)$ вьполняется интегральное неравенство

$$
\int_{\mathbb{R}^{n}}\left(\sum_{i=1}^{n} \varphi_{x_{i}} A_{i}(x, u, \nabla u)-f(x) \varphi(x)\right) d x \geqslant 0 .
$$

TЕОрема 1. Пусть $n>\alpha>1, \alpha-1>q$ и оператор L принадлежсит классу $A(\alpha)$. Тогда уравнение вида (12) не имеет обобщенных иельх положстельных суперрешений.

ОПРЕДЕЛЕНИЕ. Функцию $u(x)$ из пространства $L_{\infty, l o c}\left(\mathbb{R}^{n}\right)$ будем называть ограниченной снизу некоторой положительной константой в $\mathbb{R}^{n}$, если ess inf $u(x)$, взятьй по любому шару в $\mathbb{R}^{n}$, не меньше этой константы.

Теорема 2. Пусть $n>\alpha>1, q>\alpha-1$ и оператор L принадлежсит классу $A(\alpha)$. Тогда уравнение вида (12) не имеет ограниченных снизу полохсительной константой обобщенных иелых суперрешений.

ОПРЕДЕЛЕНИЕ. Пусть $\alpha \geqslant 1$ и $q>0$ - произвольные фиксированные действительные числа, оператор $L$ принадлежит классу $A(\alpha)$. Функцию $u(x)$ будем назьвать обобщенным иелым неотрицательным суперрешением уравнения вида (12), если она принадлежит пространству $W_{\alpha, \text { loc }}^{1}\left(\mathbb{R}^{n}\right) \cap L_{\infty, \text { loc }}\left(\mathbb{R}^{n}\right)$, неотрищательна и для произвольной неотрицательной функции $\varphi$ из пространства $\stackrel{\circ}{W}_{\alpha}^{1}\left(\mathbb{R}^{n}\right)$ вьполняется интегральное неравенство (13). 
Tеорема 3. Пусть $n>\alpha>1, n(\alpha-1) /(n-\alpha)>q>\alpha-1$, onepamop L npuнадлежит классу $A(\alpha)$ и и $(x)$ - обобщенное иелое неотрицательное суперрешение уравнения вида (12). Тогда $u(x)=0$ почти всюду в $\mathbb{R}^{n}$.

Важно отметить, что при $n>\alpha>1, q>n(\alpha-1) /(n-\alpha)$ и надлежащем $c$ радиально-симметричная функция вида

$$
u(x)=c\left(1+|x|^{\alpha /(\alpha-1)}\right)^{(1-\alpha) /(q-\alpha+1)},
$$

рассмотренная при изучении аналогичных проблем Е. Митидиери и С. И. Похожаевым, является неотрицательньм целым суперрешением уравнений вида (1), (3).

Заметим также, что теорема 2 является простьп следствием следующего утверждения.

Tеорема 4. Пусть $n>\alpha>1, q>\alpha-1$, оператор L принадлежит классу $A(\alpha) u$ $u(x)$ - обобщенное целое полохсительное суперрешение уравнения вида (12). Тогда для произвольного фиксированного $t$ из интервала $(0, \alpha-1) u \gamma=\alpha(q-t) /(q-\alpha+1)$ величина

$$
\int_{B(r)} u^{q-t} d x
$$

ecmb $O\left(r^{n-\gamma}\right)$ npu $r \rightarrow \infty$.

Здесь и далее, $B(r)$ - шар радиуса $r$ с центром в начале координат.

Утверждения, аналогичные теоремам 1-3, справедливы и при $\alpha \geqslant n$ и являются простым следствием того, что в этом случае неотрицательные решения неравенства вида $L u \leqslant 0$ с оператором $L$ из класса $A(\alpha)$ суть тождественные постоянные. Для полноты изложения приведем эти утверждения, которые непосредственно следуют из [10], [11].

ТЕОремА 5. Пусть $\alpha \geqslant n u q-$ произвольные действительные числа, а оператор L принадлежит классу $A(\alpha)$. Тогда уравнение вида (12) не имеет обобщенных иелых положительных суперрешений.

ТЕОрема 6. Пусть $\alpha \geqslant n, q>0$, оператор $L$ принадлехит классу $A(\alpha) u$ $u(x)$ - обобщенное иелое неотрицательное суперрешение уравнения вида (12). Тогда $u(x)=0$ почти всюду в $\mathbb{R}^{n}$.

ДОКАЗАТЕЛЬСТВО ТЕОРЕМЫ 1. Пусть $n>\alpha>1, \alpha-1>q$ и оператор $L$ принадлежит классу $A(\alpha)$. Предположим, что существует целое положительное суперрешение $u(x)$ уравнения вида $(12)$, и получим противоречие этому предположению. Итак, пусть $u(x)$ - целоеположительное суперрешение уравнения вида $(12), R$ и $r$ - произвольные положительные числа, $\zeta(x)$ - произвольная фиксированная функция из пространства $C^{1}(B(R))$, равная 1 на $B(r)$ и такая, что $0 \leqslant \zeta(x) \leqslant 1$. Подставим в интегральное неравенство $(13)$ в качестве пробной функции $\varphi(x)=(u(x))^{-t} \zeta^{s}(x)$, где положительные числа $s \geqslant \alpha$ и $t>\alpha-1$ будут выбраны ниже. В результате интегрирования по частям получим

$$
\begin{aligned}
& -t \int_{B(R)} \sum_{i=1}^{n} u_{x_{i}} A_{i}(x, u, \nabla u) u^{-t-1} \zeta^{s} d x+s \int_{B(R)} \sum_{i=1}^{n} \zeta_{x_{i}} A_{i}(x, u, \nabla u) u^{-t} \zeta^{s-1} d x \\
& \equiv I_{1}+I_{2} \geqslant a \int_{B(R)} u^{q-t} \zeta^{s} d x
\end{aligned}
$$


Оценим интеграл $I_{2}$ через $I_{1}$, заметив предварительно, что первое слагаемое слева в формуле (14) неположительно. Исходя из условия (7) на коэффишиенты оператора $L$, легко получаем неравенство

$$
\begin{aligned}
\left|I_{2}\right| & =\left|s \int_{B(R)} \sum_{i=1}^{n} \zeta_{x_{i}} A_{i}(x, u, \nabla u) u^{-t} \zeta^{s-1} d x\right| \\
& \leqslant \int_{B(R)} s \mathscr{K}^{1 / \alpha}\left(\sum_{i=1}^{n} u_{x_{i}} A_{i}(x, u, \nabla u)\right)^{(\alpha-1) / \alpha}|\nabla \zeta| u^{-t} \zeta^{s-1} d x .
\end{aligned}
$$

Оценивая далее подынтегральное выражение в правой части соотношения (15) по неравенству Юнга

$$
A B \leqslant \rho A^{\alpha /(\alpha-1)}+\rho^{1-\alpha} B^{\alpha}
$$

c $\rho=t / 2$,

$$
\begin{gathered}
A=u^{(1+t)(1-\alpha) / \alpha} \zeta^{s(\alpha-1) / \alpha}\left(\sum_{i=1}^{n} u_{x_{i}} A_{i}(x, u, \nabla u)\right)^{(\alpha-1) / \alpha} \\
B=s \mathscr{K}^{1 / \alpha}|\nabla \zeta| u^{(\alpha-1-t) / \alpha} \zeta^{s / \alpha-1}
\end{gathered}
$$

получим соотношение

$$
\begin{aligned}
\left|I_{2}\right| \leqslant & \frac{t}{2} \int_{B(R)} \sum_{i=1}^{n} u_{x_{i}} A_{i}(x, u, \nabla u) u^{-t-1} \zeta^{s} d x \\
& +\mathscr{K} s^{\alpha}\left(\frac{t}{2}\right)^{1-\alpha} \int_{B(R)}|\nabla \zeta|^{\alpha} u^{-t+\alpha-1} \zeta^{s-\alpha} d x
\end{aligned}
$$

Из соотношений $(14),(15),(17)$ следует неравенство

$$
\mathscr{K} s^{\alpha}\left(\frac{t}{2}\right)^{1-\alpha} \int_{B(R)}|\nabla \zeta|^{\alpha} u^{-t+\alpha-1} \zeta^{s-\alpha} d x \geqslant a \int_{B(R)} u^{q-t} \zeta^{s} d x
$$

Положим в формуле $(18) s=\alpha(t-q) /(\alpha-1-q)$ так, чтобы $(s-\alpha)(t-q) /(t-\alpha+1)=s$, и оценим его левую часть по неравенству Гёльдера с показателями $(t-q) /(t-\alpha+1)$ и $(t-q) /(\alpha-1-q)$. В результате имеет место соотношение

$$
\begin{aligned}
& \mathscr{K} s^{\alpha}\left(\frac{t}{2}\right)^{1-\alpha}\left(\int_{B(R)}|\nabla \zeta|^{\alpha(t-q) /(\alpha-1-q)} d x\right)^{(\alpha-1-q) /(t-q)} \\
& \quad \times\left(\int_{B(R)} u^{q-t} \zeta^{s} d x\right)^{(t-\alpha+1) /(t-q)} \geqslant a \int_{B(R)} u^{q-t} \zeta^{s} d x .
\end{aligned}
$$

Выберем теперь в (19) параметр $t$ из интервала $(\alpha-1, \infty)$ равным $t^{*}$ так, чтобы $\gamma=\alpha\left(t^{*}-q\right) /(\alpha-1-q)>n$. Минимизируя левую часть в неравенстве (19) по всем допустимым функциям $\zeta(x)$ указанного вида, получим

$$
\operatorname{cap}_{\gamma}\left(B(r), \mathbb{R}^{n} \backslash B(R) ; \mathbb{R}^{n}\right)\left(\mathscr{K} s^{\alpha}\left(\frac{t^{*}}{2}\right)^{1-\alpha}\right)^{\gamma / \alpha} \geqslant a \int_{B(r)} u^{q-t^{*}} d x
$$


где $\operatorname{cap}_{\gamma}\left(B(r), \mathbb{R}^{n} \backslash B(R) ; \mathbb{R}^{n}\right)$ - $\gamma$-емкость конденсатора $\left(B(r), \mathbb{R}^{n} \backslash B(R) ; \mathbb{R}^{n}\right)$. Из элементарных свойств вариационной емкости известно (см., например, [12, c. 177]), что $\gamma$-емкость конденсатора $\left(B(r), \mathbb{R}^{n} \backslash B(R) ; \mathbb{R}^{n}\right)$ есть $O\left(R^{n-\gamma}\right)$ при $\gamma \neq n, R=2 r$ и $R \rightarrow \infty$. Значит, из соотношения (20) с учетом выбора $\gamma$ и $t^{*}$ получаем, что

$$
\int_{\mathbb{R}^{n}} u^{q-t^{*}} d x=0
$$

чего быть не может. Полученное противоречие является логическим следствием нашего предположения о существовании обобщенных целых положительных суперрешений уравнения (12).

ДоКАЗАТЕЛЬСТвО ТЕОРЕМЫ 4. Пусть $n>\alpha>1, q>\alpha-1$, оператор $L$ принадлежит классу $A(\alpha)$ и $u(x)$ - обобщенное целое положительное суперрешение уравнения вида (12), $R$ и $r$ - произвольные положительные числа, $\zeta(x)$ - произвольная фиксированная функция из пространства $C^{1}(B(R))$, равная 1 на $B(r)$ и такая, что $0 \leqslant$ $\zeta(x) \leqslant 1$. Подставим в интегральное неравенство (13) в качестве пробной функции $\varphi(x)=u^{-t}(x) \zeta^{s}(x)$, а положительные числа $s \geqslant \alpha$ и $\alpha-1>t>0$ будут выбраны ниже. В результате интегрирования по частям получим неравенство

$$
\begin{aligned}
& -t \int_{B(R)} \sum_{i=1}^{n} u_{x_{i}} A_{i}(x, u, \nabla u) u^{-t-1} \zeta^{s} d x \\
& \quad+s \int_{B(R)} \sum_{i=1}^{n} \zeta_{x_{i}} A_{i}(x, u, \nabla u) u^{-t} \zeta^{s-1} d x \equiv I_{1}+I_{2} \geqslant a \int_{B(R)} u^{q-t} \zeta^{s} d x
\end{aligned}
$$

Оценивая модуль интеграла $I_{2}$ так же, как и в доказательстве теоремы 1, получим

$$
\begin{aligned}
\left|I_{2}\right| \leqslant & \frac{t}{2} \int_{B(R)} \sum_{i=1}^{n} u_{x_{i}} A_{i}(x, u, \nabla u) u^{-t-1} \zeta^{s} d x \\
& +\mathscr{K} s^{\alpha}\left(\frac{t}{2}\right)^{1-\alpha} \int_{B(R)}|\nabla \zeta|^{\alpha} u^{-t+\alpha-1} \zeta^{s-\alpha} d x
\end{aligned}
$$

Из соотношений (21), (22) следует, что

$$
\mathscr{K} s^{\alpha}\left(\frac{t}{2}\right)^{1-\alpha} \int_{B(R)}|\nabla \zeta|^{\alpha} u^{-t+\alpha-1} \zeta^{s-\alpha} d x \geqslant a \int_{B(R)} u^{q-t} \zeta^{s} d x .
$$

Положим в формуле $(23) s=\alpha(q-t) /(q-\alpha+1)$ так, чтобы $(s-\alpha)(q-t) /(\alpha-1-t)=s$, и оценим его левую часть по неравенству Гёльдера с показателями $(q-t) /(\alpha-1-t)$ и $(q-t) /(q-\alpha+1)$. В результате имеет место соотношение

$$
\begin{aligned}
& \mathscr{K} s^{\alpha}\left(\frac{t}{2}\right)^{1-\alpha}\left(\int_{B(R)}|\nabla \zeta|^{\alpha(q-t) /(q-\alpha+1)}\right)^{(q-\alpha+1) /(q-t)} \\
& \quad \times\left(\int_{B(R)} u^{q-t} \zeta^{s} d x\right)^{(\alpha-1-t) /(q-t)} \geqslant a \int_{B(R)} u^{q-t} \zeta^{s} d x .
\end{aligned}
$$


Выберем теперь в $(24)$ параметр $t$ равным произвольному фиксированному $t^{*}$ из интервала $(0, \alpha-1)$ и положим $\gamma=\alpha\left(q-t^{*}\right) /(q-\alpha+1)$. Минимизируя левую часть в неравенстве $(24)$ по всем допустимым функциям $\zeta(x)$ указанного вида, получим

$$
\operatorname{cap}_{\gamma}\left(B(r), \mathbb{R}^{n} \backslash B(R) ; \mathbb{R}^{n}\right)\left(\mathscr{K} s^{\alpha}\left(\frac{t^{*}}{2}\right)^{1-\alpha}\right)^{\gamma / \alpha} \geqslant a \int_{B(r)} u^{q-t^{*}} d x
$$

Как отмечалось выше, $\gamma$-емкость конденсатора $\left(B(r), \mathbb{R}^{n} \backslash B(R) ; \mathbb{R}^{n}\right)$ есть $O\left(R^{n-\gamma}\right)$ при $\gamma \neq n, R=2 r$ и $R \rightarrow \infty$. Следовательно, соотношение (25) влечет утверждение теоремы 4 .

ДОКАЗАТЕЛЬСТВО ТЕОРЕМЫ 3. Пусть $n>\alpha>1, n(\alpha-1) /(n-\alpha)>q>\alpha-1$, оператор $L$ принадлежит классу $A(\alpha)$ и $u(x)$ - обобщенноецелое неотрицательное суперрешение уравнения вида (12). Пусть $R, r$ и $\varepsilon$ - произвольные положительные числа, $\zeta(x)-$ произвольная фиксированная функция из пространства $C^{1}(B(R))$, равная 1 на $B(r)$ и такая, что $0 \leqslant \zeta(x) \leqslant 1$. Подставим в интегральное неравенство (13) в качестве пробной функции $\varphi(x)=(u(x)+\varepsilon)^{-t} \zeta^{s}(x)$, а положительные числа $s \geqslant \alpha$ и $\alpha-1>t>0$ будут выбраны ниже. В результате интегрирования по частям получим неравенство

$$
\begin{aligned}
& -t \int_{B(R)} \sum_{i=1}^{n} u_{x_{i}} A_{i}(x, u, \nabla u)(u+\varepsilon)^{-t-1} \zeta^{s} d x \\
& \quad+s \int_{B(R)} \sum_{i=1}^{n} \zeta_{x_{i}} A_{i}(x, u, \nabla u)(u+\varepsilon)^{-t} \zeta^{s-1} d x \\
& \equiv I_{1}+I_{2} \geqslant a \int_{B(R)} u^{q}(u+\varepsilon)^{-t} \zeta^{s} d x .
\end{aligned}
$$

Как и ранее, займемся оценкой интеграла $I_{2}$, заметив, что первое слагаемое слева в формуле (26) неположительно. В силу условия (7) на коэффициенты оператора $L$ справедливо неравенство

$$
\begin{aligned}
\left|I_{2}\right| & =\left|s \int_{B(R)} \sum_{i=1}^{n} \zeta_{x_{i}} A_{i}(x, u, \nabla u)(u+\varepsilon)^{-t} \zeta^{s-1} d x\right| \\
& \leqslant \int_{B(R)} s \mathscr{K}^{1 / \alpha}\left(\sum_{i=1}^{n} u_{x_{i}} A_{i}(x, u, \nabla u)\right)^{(\alpha-1) / \alpha}|\nabla \zeta|(u+\varepsilon)^{-t} \zeta^{s-1} d x .
\end{aligned}
$$

Оценивая далее подьнтегральное выражение в правой части формулы (27) по неравенству Юнга (16) с $\rho=t / 2$,

$$
\begin{gathered}
A=(u+\varepsilon)^{(1+t)(1-\alpha) / \alpha} \zeta^{s(\alpha-1) / \alpha}\left(\sum_{i=1}^{n} u_{x_{i}} A_{i}(x, u, \nabla u)\right)^{(\alpha-1) / \alpha}, \\
B=s \mathscr{K}^{1 / \alpha}|\nabla \zeta|(u+\varepsilon)^{(\alpha-1-t) / \alpha} \zeta^{s / \alpha-1}
\end{gathered}
$$


получим соотношение

$$
\begin{aligned}
\left|I_{2}\right| \leqslant & \frac{t}{2} \int_{B(R)} \sum_{i=1}^{n} u_{x_{i}} A_{i}(x, u, \nabla u)(u+\varepsilon)^{-t-1} \zeta^{s} d x \\
& +\mathscr{K} s^{\alpha}\left(\frac{t}{2}\right)^{1-\alpha} \int_{B(R)}|\nabla \zeta|^{\alpha}(u+\varepsilon)^{-t+\alpha-1} \zeta^{s-\alpha} d x .
\end{aligned}
$$

Из соотношений (27), (28) следует, что

$$
\mathscr{K} s^{\alpha}\left(\frac{t}{2}\right)^{1-\alpha} \int_{B(R)}|\nabla \zeta|^{\alpha}(u+\varepsilon)^{-t+\alpha-1} \zeta^{s-\alpha} d x \geqslant a \int_{B(R)} u^{q}(u+\varepsilon)^{-t} \zeta^{s} d x
$$

Перейдем в этом неравенстве к пределу при $\varepsilon \rightarrow 0$. Поскольку $t$ из интервала $(0, \alpha-1)$, в результате предельного перехода в соответствии с теоремой Лебега (см., например, [13, c. 346]), имеет место неравенство

$$
\mathscr{K} s^{\alpha}\left(\frac{t}{2}\right)^{1-\alpha} \int_{B(R)}|\nabla \zeta|^{\alpha} u^{-t+\alpha-1} \zeta^{s-\alpha} d x \geqslant a \int_{B(R)} u^{q-t} \zeta^{s} d x
$$

Положим в $(29) s=\alpha(q-t) /(q-\alpha+1)$ так, чтобы $(s-\alpha)(q-t) /(\alpha-1-t)=s$, и оценим его левую часть по неравенству Гёльдера с показателями $(q-t) /(\alpha-1-t)$ и $(q-t) /(q-\alpha+1)$. В результате имеет место неравенство

$$
\begin{aligned}
& \mathscr{K} s^{\alpha}\left(\frac{t}{2}\right)^{1-\alpha}\left(\int_{B(R)}|\nabla \zeta|^{\alpha(q-t) /(q-\alpha+1)} d x\right)^{(q-\alpha+1) /(q-t)} \\
& \quad \times\left(\int_{B(R)} u^{q-t} \zeta^{s} d x\right)^{(\alpha-1-t) /(q-t)} \geqslant a \int_{B(R)} u^{q-t} \zeta^{s} d x .
\end{aligned}
$$

Выберем теперь в (30) параметр $t$ из интервала $(0, \alpha-1)$ равным $t^{*}$ так, чтобы $\gamma=$ $\alpha\left(q-t^{*}\right) /(q-\alpha+1)>n$. Минимизируя левую часть в неравенстве (30) по всем допустимьм функциям $\zeta(x)$ указанного вида, получим соотношение

$$
\operatorname{cap}_{\gamma}\left(B(r), \mathbb{R}^{n} \backslash B(R) ; \mathbb{R}^{n}\right)\left(\mathscr{K} s^{\alpha}\left(\frac{t^{*}}{2}\right)^{1-\alpha}\right)^{\gamma / \alpha} \geqslant a \int_{B(r)} u^{q-t^{*}} d x
$$

Как уже отмечалось, $\gamma$-емкость конденсатора $\left(B(r), \mathbb{R}^{n} \backslash B(R) ; \mathbb{R}^{n}\right)$ есть $O\left(R^{n-\gamma}\right)$ при $\gamma \neq n, R=2 r$ и $R \rightarrow \infty$. Следовательно, из (31) получаем, что

$$
\int_{\mathbb{R}^{n}} u^{q-t^{*}} d x=0
$$

и, значит, $u(x)=0$ почти всюду в $\mathbb{R}^{n}$.

Автор выражает благодарность член-корреспонденту РАН С.И. Похожаеву за полезное обсуждение результатов. 


\section{СПИСОК ЦИТИРОВАННОЙ ЛИТЕРАТУРЫ}

[1] Gidas B., Spruck J. Global and local behaviour of positive solutions of nonlinear elliptic equations // Comm. Pure Appl. Math. 1981. V. 34. P. 525-598.

[2] Ni W.-M., Serrin J. Non-existence theorems for quasilinear partial differential equations // Rend. Circ. Mat. Palermo (2). 1985. V. 5. P. 171-185.

[3] Bidaut-Veron M. F. Global and local behaviour of solutions of quasilinear equations of Emden-Fowler type // Arch. Rational Mech. Anal. 1989. V. 107. P. 293-324.

[4] Clement Ph., Monasevich R., Mitidieri E. Positive solutions of quasilinear systems via blow-up // Comm. Partial Differential Equations. 1993. V. 18. P. 2071-2106.

[5] Berestycki H., Capuzzo Dolcetta J., Nirenberg L. Superlinear indefinite elliptic problems and nonlinear Liouville theorems // Topol. Methods Nonlinear Anal. 1995. V. 4. P. 59-78.

[6] Mitidieri E. Nonexistence of positive solutions of semilinear elliptic systems in $\mathbb{R}^{n}$ // Differential Integral Equations. 1996. V. 9. P. 465-479.

[7] Курта В. В. Некоторые вопросы качественной теории нелинейных дифференциальных уравнений второго порядка. Дисс. ... д. ф..-м. н. М., 1994.

[8] Курта В. В. Некоторые вопросы качественной теории нелинейных дифференциальных уравнений второго порядка. Автореферат дисс. ... д. ф.-м. н. М., 1995.

[9] Курта В.В. К вопросу об отсутствии целых положительных решений у полулинейных эллиптических уравнений // УМН. 1995. Т. 50. №4. С. 127.

[10] Миклюков В. М. Об одном новом подходе к теореме Бернштейна и близким вопросам уравнений типа минимальной поверхности // Матем. сб. 1979. Т. 108 (150). № 2. С. 268-289.

[11] Миклюков В. М. Емкость и обобщенный принцип максимума для квазилинейных уравнений эллиптического типа // Докл. АН СССР. 1980. Т. 250. №6. С. 1318-1320.

[12] Гольдштейн В. М., Решетняк Ю. Г. Введение в теорию функций с обобщенными производными и квазиконформные отображения. М.: Наука, 1983.

[13] Колмогоров А. Н., Фомин С. В. Элементы теории функций и функционального анализа. М.: Наука, 1989.

Институт прикладной математики и механики НАН Украины,

E-mail: kurta@iamm.ac.donetsk.ua 